\title{
CFD modelling of heat transfer and pressure drops for nanofluids through vertical tubes in laminar flow by Lagrangian and Eulerian approaches
}

\author{
M. Mahdavi, M. Sharifpur", J.P. Meyer \\ Department of Mechanical and Aeronautical Engineering, University of Pretoria, \\ Private Box X20, Hatfield, 0028, Pretoria, South Africa.
}

\begin{abstract}
Nanofluids consist of liquid and solid (nanoparticles), therefore, they can be classified as two-component flow, which brings up different approaches for simulation purposes. In this study, heat transfer and hydro-dynamic features of nanoparticles in a laminar nanofluid flow in a vertical tube are investigated numerically via Lagrangian and Eulerian approaches. Discrete Phase Model (DPM) in Lagrangian approach simulates the motion of particles through base flow with force balance equation, therefore, no needs empirical correlations at least for the thermo-physical properties (which they are not universal and change for different fluids and/or nanoparticles). Although, general empirical or analytical correlations are needed for some interactions between solid and liquid such as Thermophoresis, Brownian and clustering effects, but they are not that extensive and can be employed in most of the cases. Mixture model in Eulerian approach provides more reliable results, but it highly depends on the accuracy of the correlations for the thermo-physical properties of the nanofluid. In present study, three common types of nanofluids consist of Alumina, Zirconia and Silica nanoparticles (up to $2.76 \%$ of volume fraction) are studied and the results are compared with experimental works. Numerical simulations indicate that the findings are in good agreement with the measured heat transfer coefficient for DPM. Consequently, DPM can be highly recommended for simulation study due to the strength and simplicity. It has been also observed that the effects of nanoparticles in each computational cell need to be distributed to the other neighbourhood cells. Pressure losses results predicted by DPM were found reliable for volume fraction less than $3 \%$, no matter the types of nanoparticles or diameter. DPM velocity profiles show that the slip velocity between nanoparticles and base flow is not negligible.
\end{abstract}

Keywords: nanofluids, laminar, vertical tube, DPM, Mixture model, CFD 


\section{Introduction}

Nanofluids have shown higher heat transfer performance in comparison with conventional heat transfer fluids, therefore, they have interested by many researchers in recent years. Nanoparticles are easily suspended in conventional heat transfer fluids (as base fluids) to produce stable nanofluids in comparison with micro-particles. On the other hand, the advantage of nanoparticles comparing to other particles is higher surface area per volume which clearly enhance heat transfer rate. In addition, random movement of ultrafine particles can be the other main reason of the heat transfer enhancement. Experimental findings explain that the heat transfer improvement by nanofluids can vary from small percentage [1] to a few times bigger than the base fluid (without particles) [2]. Some reviews of experimental work in convective laminar nanofluid flow are available in literature [3, 4].

Wen and Ding [5] showed that nanoparticles influence heat transfer enhancement extensively in laminar flow in a tube, especially in entrance regain. They also mentioned that particle movement can be one of the main reasons for higher heat exchange.

Liu and $\mathrm{Yu}$ [6] found that non-uniform radial distribution of particles is mostly affected by particle migration, especially in the boundary layer region. They explained that the interaction between nanoparticles and fluid is remarkable and as a result heat transfer can be sensibly enhanced in higher Reynolds number, even in laminar flow.

Utomo et al. [7] explained that particles migration mainly caused by Brownian effects and thermophoresis has negligible impacts on increase of heat transfer characteristics. Nanoparticles originally aim to enhance heat transfer rate by increasing the effective thermal conductivity, while the negative impacts of adding particles to fluid flow on pressure drops cannot be neglected. It has been observed that pressure drop in $\mathrm{SiO}_{2}$ nanofluid with $5 \%$ volume fraction can exceed 10 times [8], although small changes in friction factor have been reported for Alumina nanofluid in lower particle loading [9].

Because of the existence of solid and liquid phases in the nanofluid flow, it can be classified as two-component flow, which brings up different approaches of multiphase flow for study purposes. Considering nanoparticles as a secondary phase in flow has been investigated by some researchers [10, 11]. The multiphase model employed in most nanofluid studies is Mixture model from Eulerian-Eulerian approach which is relied more on nanofluid thermophysical properties gained by empirical correlations. The interaction between nanoparticles 
and liquid phases is simulated with capturing the slip velocity between particles and base flow. It is believed that Mixture model may be able to include the effects of slip velocity [12]. Also, Hwang et al. [1] explained slip velocity between fluid and nanoparticles as the strong mechanism for particle migration and flattened velocity at the centre of tube. They used mixture thermo-physical properties with considering Brownian and Thermophoresis diffusion terms. Kalteh et al. [13] employed Eulerian two-phase model to simulate laminar nanofluid in a micro-channel. They argued that the slip velocity and temperature difference between nanoparticles and base fluid is not noticeable and uniform distribution of nanoparticles in flow field is expected. Their results for heat transfer enhancement showed more than single phase model and pressure drops were barely influenced by nanoparticle loading. Lotfi et al. [14] compared Mixture model and two-phase Eulerian model with single phase model for convective water- $\mathrm{Al}_{2} \mathrm{O}_{3}$ nanofluid in horizontal tubes and concluded that Mixture model has better Nusselt number predictions.

Lagrangian with Discrete Phase Model (DPM) which is an approach of multi-phase modelling, simulates motion equation of each particle in flow field. In the DPM the interaction between particle and fluid occurs by the forces acting on particles. The main important phenomena in heat transfer and hydrodynamic behaviour of nanoparticles in a nanofluid flow can be Brownian motion, thermophoresis force, forming a liquid layer around a particle, clustering and interaction forces [12, 15, 16, 17, 18, 19]. Jang and Chio [15] proposed a theoretical model to capture the impacts of nanoparticles on effective thermal conductivity. They stated that Brownian motion is the significant mechanism on heat transfer characteristic of nanofluid. The induced collision between nanoparticles was also found negligible in comparison with other modes of collision in nanofluid. They concluded that effective thermal conductivity caused by thin liquid layer around particle play a key role in increase of heat transfer rate, especially in smaller size of nanoparticles, shown by Yu and Choi [20] as well.

Schio et al. [18] concluded that the thermal influences of Brownian and thermophoresis diffusion on nanofluid may not be imperceptible and there is a distinct non-homogeneity in the flow.

A few studies have been done in the field of laminar nanofluid flow by DPM. He et al. [21] and Bianco et al. [22] were pioneer in modelling nanoparticles as discrete phase. The former employed the particles interaction source term only in momentum equation, but the latter 
used both momentum and energy source terms in equations. He et al. stated that heat transfer enhancement is mainly affected by thermal conductivity and other forces like Brownian and thermophoresis have small impacts. They indicated that the heat transfer results by DPM are in good agreement with experiments. Tahir and Mital [23] carried out a numerical simulation of nanofluids in a laminar tube flow by DPM and found good results comparing to experimental data. They considered both particle momentum and energy source terms in equations and discussed only the results of heat transfer characteristics.

In this research, the abilities and weaknesses of Mixture and DPM models are discussed and compared with some experimental studies. The main advantage of DPM comparing to Mixture model is the thermo-physical properties of nanofluid which no needs to be obtained experimentally. On the other hand, lack of universal properties for nanofluids is an unsolved problem for Mixture model. Due to lack of information on dynamic behaviour of nanoparticles in flow field, it seems important to investigate the power of DPM in modelling nanofluids, as a few studies have been done in this area. Both Mixture model and DPM results are assessed with available experiments by Rea et al. [24] and Zhang [25] for three types of nanofluids consist of Alumina, Zirconia and Silica nanoparticles. Then, the results predicted by two models are compared and discussed.

\section{Numerical simulations}

Based on experimental works from literature, two different diameter sizes of vertical tubes are simulated in this study, with the same length of $1 \mathrm{~m}$. Rea et al. [24] used stainless steel tube with ID $4.5 \mathrm{~mm}$, and Zhang [25] with $5.537 \mathrm{~mm}$. Both the tubes are encountered constant heat flux on the outside of the tubes. Three types of nanoparticles as nanofluids are injected through the tubes consist of $\mathrm{Al}_{2} \mathrm{O}_{3}$ and $\mathrm{ZrO}_{2}$ by [24] and $\mathrm{SiO}_{2}$ by [25]. In all the tests, Reynolds number is below 2000 and therefore flow is laminar. Two multiphase models for simulation, namely Mixture model and DPM are described as follows.

A simple curve fitting were carried out to choose proper polynomial thermo-physical properties of water as the base fluid in this research which are available in heat transfer books and literature [26]. They are described in Table 1 with thermo-physical properties of nanoparticles. 
Table 1. Thermo-physical properties of water and the nanoparticles

\begin{tabular}{|c|c|c|c|c|c|}
\hline & $\rho\left(k g / m^{3}\right)$ & $c_{p}(J / k g . k)$ & $\kappa(W / m . k)$ & $\mu(k g / m . s)$ & $d_{p}(n m)$ \\
\hline water & $\begin{array}{l}765.33+1.8142 \times \mathrm{T} \\
-0.0035 \times \mathrm{T}^{2}\end{array}$ & $\begin{array}{l}(28.07-0.2817 \times \mathrm{T} \\
+0.00125 \times \mathrm{T}^{2} \\
-2.48 \mathrm{e}-6 \times \mathrm{T}^{3} \\
\left.+1.857 \mathrm{e}-9 \times \mathrm{T}^{4}\right) \times 1000\end{array}$ & $\begin{array}{l}-0.5752+0.006397 \times \mathrm{T} \\
-8.151 \mathrm{e}-6 \times \mathrm{T}^{2}\end{array}$ & $\begin{array}{l}0.0967-8.207 \mathrm{e}-4 \times \mathrm{T} \\
+2.344 \mathrm{e}-6 \times \mathrm{T}^{2} \\
-2.244 \mathrm{e}-9 \times \mathrm{T}^{3}\end{array}$ & - \\
\hline $\mathrm{Al}_{2} \mathrm{O}_{3}$ & 3920 & 880 & 36 & - & 50 \\
\hline $\mathrm{ZrO}_{2}$ & 5600 & 418 & 2 & - & 50 \\
\hline $\mathrm{SiO}_{2}$ & 2200 & 745 & 1.38 & - & 20 \\
\hline
\end{tabular}

Temperature is in terms of kelvin.

\subsection{Mixture model}

Slip velocity between nanoparticles and base fluid is assumed small in this model and at the same time, there is a strong coupling between them. Only one momentum and one energy equation are solved for entire nanofluid mixture and each phase has its own velocity and fraction in each computational cell. More details about the governing equations of this model can be found in previous works $[14,27]$. In the absence of mass exchange in the steady state flow, governing equations consist of mass, momentum and energy are derived for each phase and eventually the linear summation of these presents the final equations for Mixture model:

Mixture continuity:

$$
\nabla \cdot\left(\rho_{m} \vec{V}_{m}\right)=
$$

Mixture conversation of momentum:

$$
\nabla \cdot\left(\rho_{m} \vec{V}_{m} \vec{V}_{m}\right)=-\nabla P_{m}+\nabla \cdot \tau_{m}+\rho_{m} g-\nabla \cdot \sum_{k=1}^{2} \alpha_{k} \rho_{k} \vec{V}_{k m} \vec{V}_{k m}
$$

where $\vec{V}_{m}, \tau_{m}$ and $\alpha$ are mixture velocity, shear stress tensor and volume fraction, respectively. The drift velocity, $\vec{V}_{k m}$, is defined as: 


$$
\begin{aligned}
& \vec{V}_{k m}=\vec{V}_{k}-\vec{V}_{m} \\
& \vec{V}_{m}=\frac{\sum_{k=1}^{2} \alpha_{k} \rho_{k} \vec{V}_{k m}}{\rho_{m}} \\
& \rho_{m}=\sum_{k=1}^{2} \alpha_{k} \rho_{k}
\end{aligned}
$$

For two components flow field, the connection between drift and slip or relative velocity is:

$$
\vec{V}_{p m}=V_{\text {relative }}-\frac{\alpha_{p} \rho_{p}}{\rho_{m}} V_{\text {relative }}=\frac{\alpha_{c} \rho_{c}}{\rho_{m}} V_{\text {relative }}
$$

$\alpha_{c}$ and $\rho_{c}$ are continues phase (here is water) volume fraction and density, respectively. A constitutive equation is needed here to complete the Mixture model. Hence, the slip velocity can be obtained from the following algebraic equation from Manninen et al. [28]:

$$
V_{\text {relative }}=\frac{\rho_{p} d_{p}^{2}}{18 \mu_{c} f_{d}} \frac{\left(\rho_{p}-\rho_{m}\right)}{\rho_{p}}\left[\vec{g}-\left(\vec{V}_{m} \cdot \nabla\right) \vec{V}_{m}-\frac{\partial \vec{V}_{m}}{\partial t}\right]
$$

The drag function $f_{d}$ can be calculated from drag coefficient, $C_{D}$, as [29]:

$$
f_{d}=C_{D} \frac{\operatorname{Re}_{p}}{24}=1+0.15 \operatorname{Re}_{p}^{0.687} \quad \operatorname{Re}_{p} \leq 1000
$$

Mixture conservation of energy:

$$
\nabla \cdot\left(\rho_{m} \vec{V}_{m} H_{m}\right)=-\nabla \cdot\left(q_{m}\right)-\nabla \cdot \sum_{k=1}^{2} \alpha_{k} \rho_{k} \vec{V}_{k m} H_{k}
$$

$H_{m}$ and $q_{m}$ are mixture enthalpy and heat flux due to conduction. Distribution of particle phase through continues phase is determined from volume fraction equation, simply derived from particle phase continuity with constant density:

$$
\nabla \cdot\left(\alpha_{p} \vec{V}_{m}\right)=-\nabla \cdot\left(\alpha_{p} \vec{V}_{p m}\right)
$$


The particle Reynolds number is defined based on relative velocity between particle and fluid phases, $\operatorname{Re}_{p}=\frac{d_{p} \rho_{c} V_{\text {relative }}}{\mu_{c}}$ where $d_{p}, \rho_{p}$ and $\mu_{m}$ are particle diameter, particle density and mixture viscosity, respectively. The key parameters in Mixture model are nanofluid thermophysical properties which are mostly employed from experimental correlations. Since the nanofluid thermo-physical properties were available $[24,25]$, they are borrowed to implement in mixture properties.

\subsection{Discrete Phase Model}

In this model, nanoparticles are tracked as a large number of particles and all the energy or force interactions are introduced as momentum or energy source terms in the governing equations. The rest of the Navier Stokes equations are treated as usual for the base fluid. Differential form of force balance equation is applied for a particle suspended in the flow to calculate the trajectory of the particle.

Interaction forces acting between particles and base fluid are presented as: drag force, Brownian Force by the random motion of particles through the base fluid, gravity force, Thermophoretic force due to a temperature gradient and especially close to the wall. These interaction forces are presented as a momentum source term in momentum equation. The motion equation of single nanoparticle is simply defined as:

$$
\frac{d V_{p}}{d t_{p}}=\frac{18 \mu_{c}}{\rho_{p} d_{p}^{2}} \frac{C_{D} \operatorname{Re}_{p}}{24}\left(\vec{V}_{c}-\vec{V}_{p}\right)+\frac{\vec{g}\left(\rho_{p}-\rho_{c}\right)}{\rho_{p}}+\vec{F}_{o t h e r}
$$

where $t_{p}$ and $\vec{F}_{\text {other }}$ are particle motion time from one cell to the next one in Lagrangian reference frame, and the other forces acting on a particle per unit mass of the particle. Since the tube used in this study is vertical, gravity force may play a key role in particle motion, although Rea [30] noted that no sedimentation was observed in laminar force flow. Nonetheless, nanoparticles are still able to cause pressure drops in base fluid duo to gravity without sedimentation.

Drag coefficient for smooth spherical particle is introduced as:

$$
C_{D}=a_{1}+\frac{a_{2}}{\operatorname{Re}_{p}}+\frac{a_{3}}{\operatorname{Re}_{p}^{2}}
$$


The constant values $a_{i}$ are available for a wide range of Reynolds number [31]. Because of the nanoparticles are ultrafine, $\operatorname{Re}_{\mathrm{p}}<<1$ and $a_{1}=a_{3}=0$ and $a_{2}=24$ gives $C_{D}=\frac{24}{\operatorname{Re}_{p}}$. $\operatorname{Re}_{\mathrm{p}}$ (particle Reynolds number) was defined in the previous section. Thermophoretic velocity is presented as the following:

$$
\begin{aligned}
& V_{T}=-0.26 \frac{\mu_{c}}{\rho_{c}} \frac{\kappa_{c}}{2 \kappa_{c}+\kappa_{p}} \frac{\nabla T}{T} \\
& F_{T}=-3 \pi d_{p} \mu_{c} V_{T}=-D_{T} \frac{\nabla T}{T} \\
& D_{T}=0.78 \frac{\pi \mu_{c}^{2} d_{p}}{\rho_{c}} \frac{\kappa_{c}}{2 \kappa_{c}+\kappa_{p}}
\end{aligned}
$$

Thermophoretic force was derived from Stokes's drag force law. $V_{T}, F_{T}$ and $D_{T}$ are thermophoretic velocity, force and coefficient, respectively. $\kappa_{p}$ is particle thermal conductivity and $\rho_{c}, \mu_{c}$ and $\kappa_{c}$ are fluid density, viscosity and thermal conductivity. Brownian force is presented as [33]:

$$
\begin{aligned}
& S=216 \frac{K_{B} \mu_{c} T}{\pi^{2} \rho_{p}^{2} d_{p}^{5} C_{c}} \\
& F_{B_{i}}=\psi_{i} \sqrt{\frac{\pi S}{\Delta t}}
\end{aligned}
$$

where $F_{B_{i}}, \psi_{i}, C_{c}$ and $\Delta t$ are random Brownian force, Gaussian noise function with zero mean value, Cunningham correction (to modify the possibility of slip condition at particle surface duo to non-continuum condition) and Lagrangian time step, respectively.

Amount of heat transfer exchange is easily calculated from energy balance for each particle at the inlet to the outlet of a cell. This exchange will be implemented as an energy source term in continues phase energy equation. This source term is simply calculated from energy balance inside a computational cell as:

$$
Q_{s}=\dot{m}_{p} c_{p_{p}}\left(T_{p_{\text {in }- \text { cell }}}-T_{p_{\text {out cell }}}\right)
$$


Since there is no mass exchange, particle flow rate $\dot{m}_{p}$ in each cell will be conserved. $c_{p_{p}}$, $T_{p_{\text {ĭ }}}$ and $T_{p_{\text {out }}}$ are particle specific heat, particle temperature at the inlet and outlet of a cell, respectively. The exchanged heat at the surface of a particle is calculated as following to find $T_{p_{\text {it }}}$ and $T_{p_{\text {out }}}$.

$m_{p} c_{p_{p}} \frac{d T_{p}}{d t}=h A_{p}\left(T_{c}-T_{p}\right)$

With definition of Biot number for a particle as $B i=\frac{h d_{p}}{6 \kappa_{p}}$, it can be expected to have uniform temperature inside a particle in each Lagrangian time step duo to very small amount of Biot number. Heat transfer coefficient around a spherical particle can be estimated from the following correlation [32]:

$$
\frac{h d_{p}}{\kappa_{c}}=2+0.6 \operatorname{Re}_{p}^{0.5} \operatorname{Pr}^{1 / 3}
$$

\section{Numerical method and geometry model}

Both 2D axisymmetric and 3D models have been employed in this study and the results showed that it is crucial to use 3D model in laminar nanofluid flow, especially with the presence of gravity in Lagrangian approach. It can be discussed that 2D axisymmetric is only able to capture radial migration of nanoparticles inside the fluid, while the difference between 3D model and 2D axisymmetric simulation results prove that nanoparticles migrate from the wall both radially and tangentially. Also, simulations have shown that gravity cannot be neglected when DPM is used and absence of gravity force underestimates pressure drops in vertical tubes. The CFD software employed in this research was FLUENT 15 [33] to solve the governing equations, using control volume approach. SIMPLE method was employed to couple pressure and velocity in equations, QUICK scheme for volume fraction and Second Order Upwind for interpolating other parameters. Both the pressure interpolation schemes, i.e. Standard and Linear have shown the same results for pressure drops in DPM. It is noted that PRESTO! interpolation scheme is not recommended for pressure discretization in DPM. Since pressure values are needed on the faces of each control volume to discretise in momentum equation, Standard and Linear schemes evaluate pressure on the face based on cell values at neighbourhoods, with the assumption of zero pressure gradient at the wall. 
While the PRESTO! scheme estimates pressure on the face via continuity balance based on staggered control volume with non-zero pressure gradient at the wall [33, 34]. Using PRESTO! scheme provides unreal pressure distribution results at the tube entrance with DPM. It may come from the solution method of DPM, especially with high number of particles in each computational cell (order of $10^{10}$ ). Also, nanoparticles are injected in the flow at entrance uniformly, while boundary layer starts forming in base flow and produce slip velocity between base flow and nanoparticles at the vicinity of the wall. The number of particles in each computational cell, named parcel, is order of $10^{10}$. It can probably produce a considerable amount of source term in momentum equation and pressure drops predicted by PRESTO! scheme, because of the solution method of this scheme at the vicinity of the wall. On the other hand, it can be explained also with the source term added to the momentum equation as:

$$
F_{s}=\sum\left(\frac{18 \mu_{c}}{\rho_{p} d_{p}^{2}} \frac{C_{D} \operatorname{Re}_{p}}{24}\left(\vec{V}_{p}-\vec{V}_{c}\right)+\vec{F}_{\text {other }}\right) \dot{m}_{p} \Delta t
$$

$\dot{m}_{p}$ and $\Delta t$ are particle flow rate in each computational cell and integral time step, respectively. Therefore, the source term can produce huge value due to incorporate of $1 / d_{p}^{2}$ in the equation. On the other hand there is a noticeable difference between particle and flow velocities at the entrance, because of undeveloped particle and flow boundary layer. In spite of Standard or linear interpolation schemes, PRESTO! scheme interpolates pressure at the face centre of a cell (it can be called face pressure instead of cell pressure) where particles are interred the cell. Large difference between particle and flow velocities (based on the above source term) at the entrance produces a large body force at the face of a cell (staggered grid) which make unreal large amount of pressure interpolated by PRESTO! scheme. From the momentum source term, the only way to overcome this problem seems to provide very small time step. Particle Lagrangian time step depends on the size of the cell that a parcel passes, smaller the size means shorter in time step. Simulation showed some improvement with very fine mesh at the entrance with PRESTO! Scheme, but the geometry requires grids with the size similar to DNS to overcome this issue completely, which is not the main goal of this study.

A nanoparticle in each parcel is representative of the entire nanoparticles on that parcel and heat transfer and motion equations are solved only for this nanoparticle and extended to 
others. Therefore, the distribution effects of these nanoparticles on parcels in neighbourhood are not usually applied in the equations, which can be called point-particle approach. For this aim, Node based averaging method is employed to consider those influences from Lagrangian frame onto Eulerian field. Gaussian distribution function is used to interpolate the neighbouring parcel's impacts on the centroid parcel, as the following $[33,35]$ :

$$
\begin{aligned}
& \bar{\theta}_{\text {parcel }}=\sum N_{\text {particle }} G_{w} \theta_{\text {particle }} \\
& G_{w}=\left(\frac{a}{\pi}\right)^{3 / 2} \exp \left(-a \frac{\left|x_{\text {parcel }}-x_{\text {particle }}\right|^{2}}{\Delta x^{2}}\right)
\end{aligned}
$$

where $\bar{\theta}_{\text {parcel }}, N_{\text {particle }}, G_{w}, \theta_{\text {particle }}, \Delta x, x_{\text {parcel }}$ and $x_{\text {particle }}$ are particle variable affected by nodes in neighbourhood (which are 26 cells for structured quad mesh in this research), number of particles in the parcel, Gaussian weight function, particle variable in the node, characteristic length of the cell, parcel location in neighbourhood and particle location, respectively. $a$ is a constant which changes the width of Gaussian function. The number of particles in each parcel can be calculated as:

$$
N_{\text {particle }}=\dot{m}_{p} \frac{\Delta t}{m_{p}}
$$

Particle variables $\theta_{\text {particle }}$ are achieved from DPM equations and then the new amount of variables $\bar{\theta}_{\text {parcel }}$ affected by neighbouring cells will be calculated through equation (22). This new value corporates in the source terms of the base fluid equations. The simulations show that the results by DPM with Node based averaging are in better agreement with experiments and the residuals has indicated improved convergence than without Node based averaging method. It is believed that existence of interpolation operators like Gaussian kernel function can improve and accelerate the numerical solution [35, 36], especially in the case of dense number of nanoparticles in each computational cell. Grid study proves that a structured mesh with higher uniformity can provides more precise results by DPM than other meshes. However, higher number of cells is needed in modelling nanofluid by DPM comparing to other laminar single phase flow in the same geometry. The final number of grids for this study was chosen as 16 radially, 58 tangentially and 600 longitudinally $(16 \times 58 \times 600)$, which shown in Figure 1 . The closets node to the wall is almost $1 \times 10^{-4} \mathrm{~m}$ which is less than $5 \%$ of 
tube radius. It is noted that the simulation results achieved by this mesh and even many times more than this without Node based averaging method are not in agreement with experimentations. Boundary conditions for DPM consist of: similar uniform velocity and temperature at the inlet for both particles and base flow, particle mass flow rate at the inlet based on particle volume fraction (it is important to make sure particles are uniformly distributed at the inlet), constant heat flux over the external surface of the tube, fully developed condition at the outlet and no-slip condition at the wall of tube. It is noted that inlet conditions for particles are equivalent boundary and initial conditions for particles in Lagrangian frame. Also, wall boundary conditions differ for the particles than base flow. Two main possibilities are that they can either rebound off the wall or stick to the wall. Simulation results showed that none of the mentioned conditions happen for particles. It means that particles never reach the wall or meet the wall conditions. Mixture model B.C. is similar to base flow conditions in DPM. For particle phase with the same velocity as primary phase, the proper amount of volume fraction is assigned.

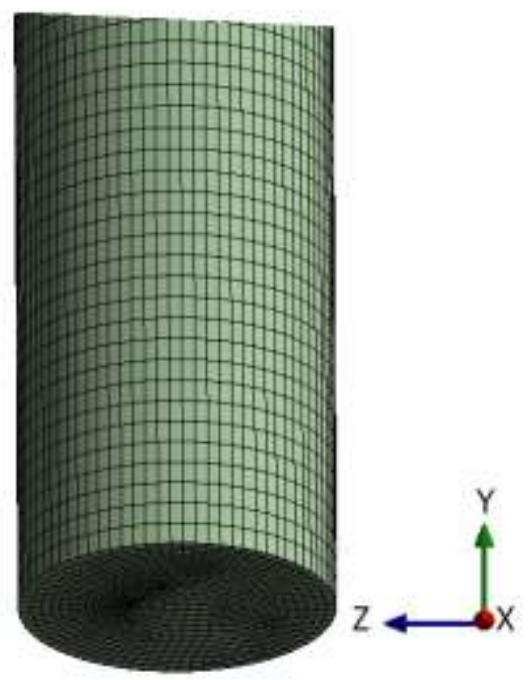

Figure 1. Generated mesh for CFD study with Y as vertical direction

\section{Results and discussion}

At the first step, the amount of parameter $a$ in Gaussian distribution function needs to be chosen. Kaufmann et al. [37] successfully used $a=6$ in their study. Simulation results have proved that any changes in $a$ influences mostly particles concentration field and it has small impacts on pressure drops and heat transfer coefficient. The effects of $a$ on Alumina nanofluid with volume fraction $2.76 \%$ at the outlet of the tube can be seen in Figure 2. It is true that the larger amount of $a=6$ provides higher uniformity than smaller one, but still the 
presence of concentration gradient is obvious in all of them. Also, the same pattern of distribution can be observed for different ranges of $a$. More visual observations of concentration distribution for nanofluid are needed to find the optimum amount of $a$. However, $a=6$ was chosen for all the simulations here similar to Kaufmann et al. [37].

The importance of gravity can be seen in figure 3. When gravity is excluded from DPM, the pressure drops predicted by simulations are close to pressure drops with pure water, while DPM predicts more than twice as pressure loss with pure water. Hence, considering no gravity in DPM may produce errors up to $200 \%$ in vertical laminar flow.

Dispersion of Alumina and Zirconia nanoparticles at the outlet section of the tube are illustrated in Figure 4.

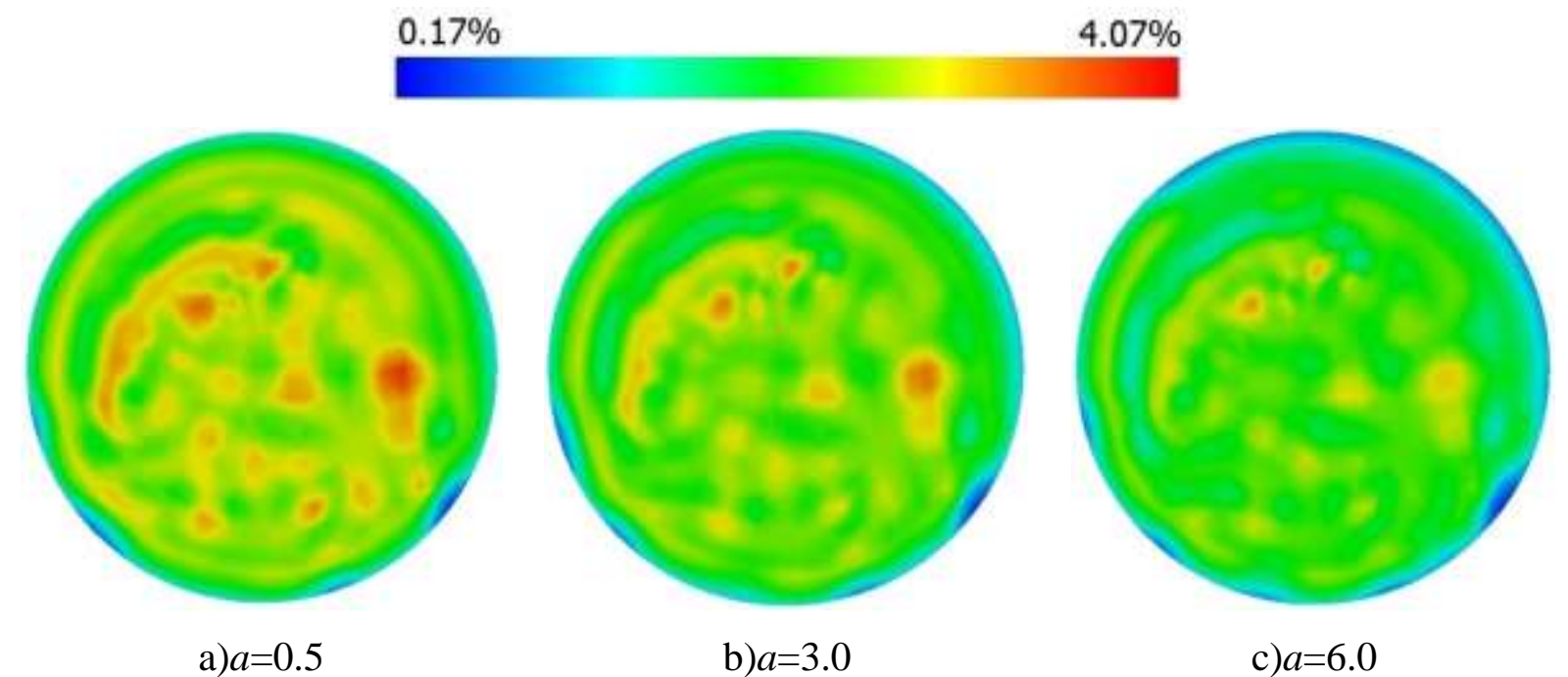

Figure 2. Effects of parameter $\boldsymbol{a}$ in Gaussian function on concentration distribution of Alumina nanofluid with $\alpha=2.76 \%$ at the outlet of the tube at $R e=1131, T_{i n}=22.9$ and $q=18629 \mathrm{~W} / \mathrm{m}^{2}$. 


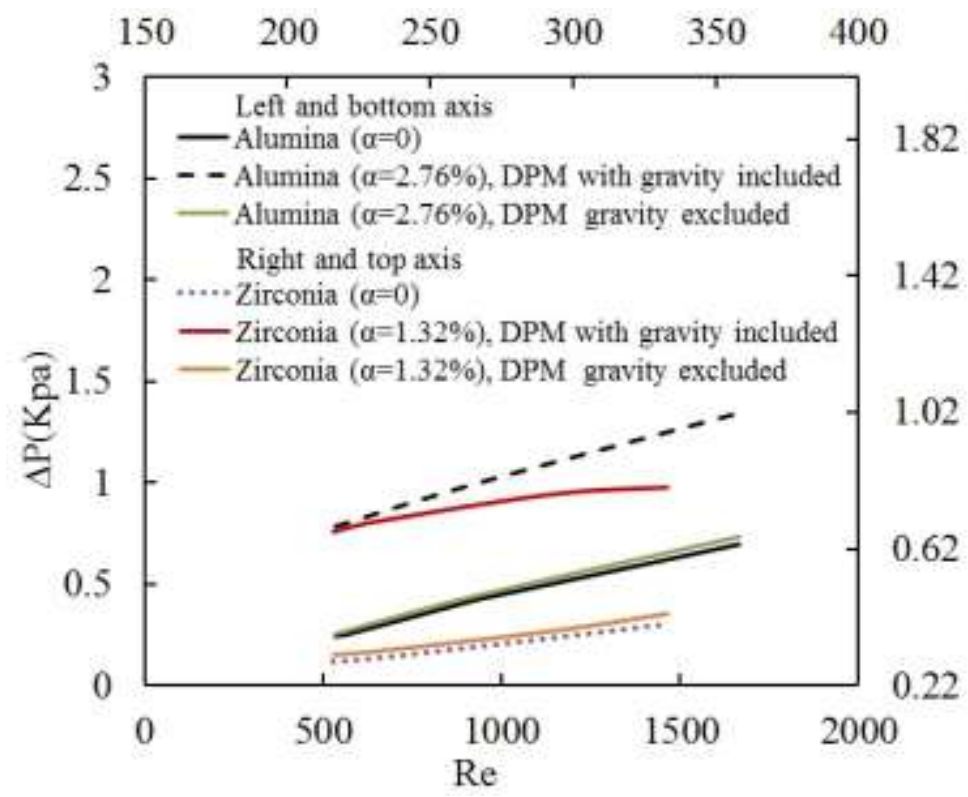

Figure 3. Effects of presence of gravity in DPM simulations for Alumina and Zirconia nanofluids.

The pictures show the similar pattern of distribution for both Alumina and Zirconia nanofluids in different volume fractions. The percentage range of volume fraction in both nanoparticles with various particle loadings demonstrates that nanoparticles follow almost uniformly the base flow in lower volume fractions than higher concentration, no matter the type of the nanoparticles. Concentration of nanoparticles rises in cells with increase in particle loading, especially in an area slightly far from the wall and centroid region of tube, clearly seen in Figure 4d. Again, the types of the nanoparticles have shown no impacts on dispersion of particles on the tube.

Figure $4 \mathrm{~d}$ also proves that the possibility of clustering may accelerate with growth in nanoparticles volume fraction. One of the reasons for no symmetry in particle distribution can be considering Brownian motion and Thermophoresis at the same time. Brownian motion happens everywhere in cross section and Thermophoresis is strong close to the wall. The combination of both may disturb the cross sectional concentration distribution. On the other hand, Mixture model results have provided more uniform distribution of concentration than DPM for any types of nanoparticles and particle loadings. It can be pointed out that Mixture model treats nanofluid as a homogenous mixture with no relative velocity between solid and liquid parts. 


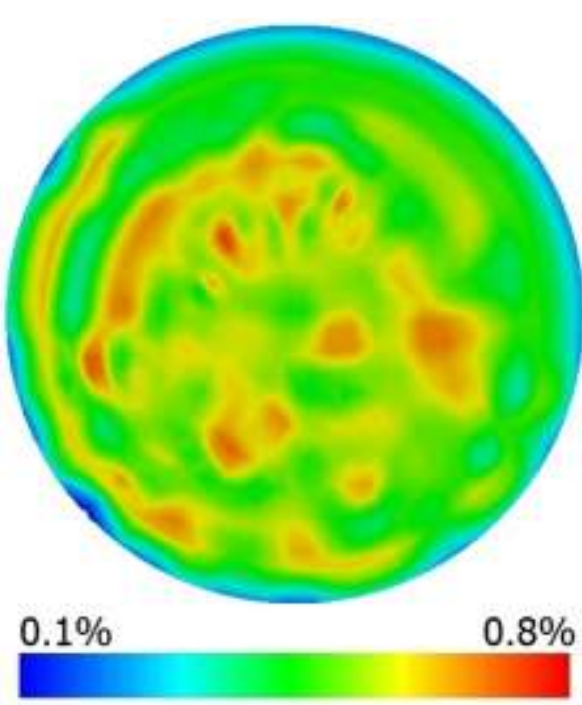

a) $0.65 \%$ Alumina nanofluid, $\mathrm{Re}=1797$, $\mathrm{T}_{\text {in }}=21.7$ and $\mathrm{q}=18633 \mathrm{~W} / \mathrm{m}^{2}$

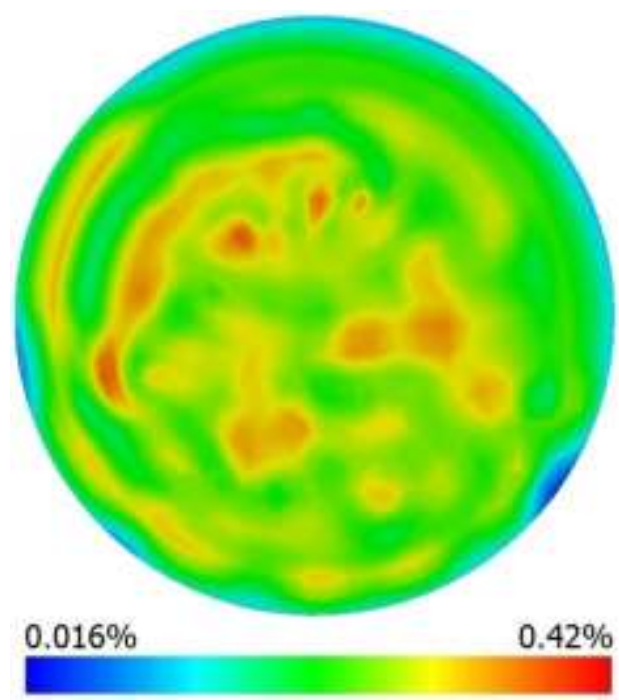

c) $0.32 \%$ Zirconia nanofluid, $\mathrm{Re}=356, \mathrm{~T}_{\mathrm{in}}=22$ and $\mathrm{q}=19359 \mathrm{~W} / \mathrm{m}^{2}$

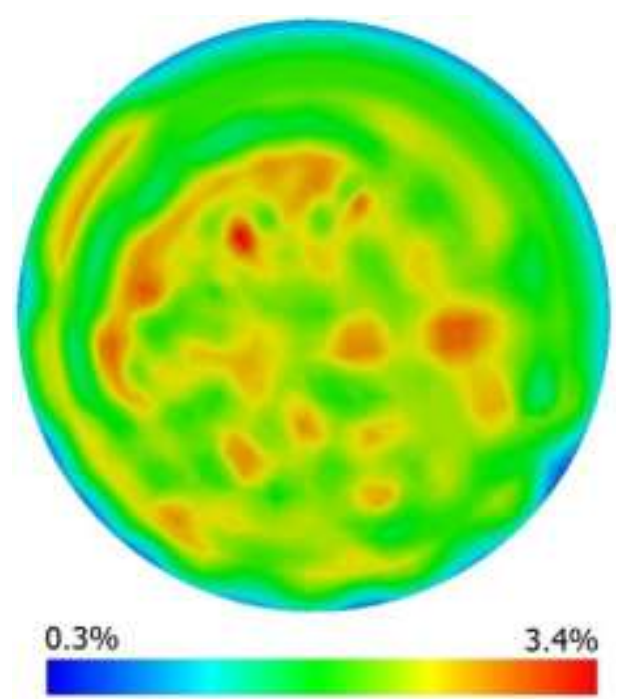

b) $2.76 \%$ Alumina nanofluid, $\mathrm{Re}=1666$, $\mathrm{T}_{\mathrm{in}}=23.1$ and $\mathrm{q}=25306 \mathrm{~W} / \mathrm{m}^{2}$

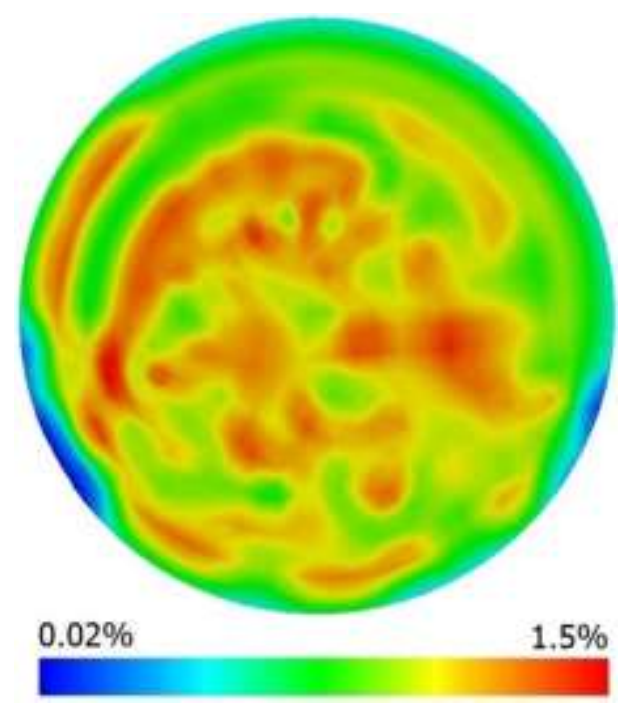

d) $1.32 \%$ Zirconia nanofluid, $\operatorname{Re}=293, \mathrm{~T}_{\mathrm{in}}=22$ and $\mathrm{q}=16308 \mathrm{~W} / \mathrm{m}^{2}$

Figure 4. Distribution of nanoparticles volume fraction at the outlet for Alumina and Zirconia nanofluids.

Heat transfer and hydrodynamic characteristics of laminar nanofluid in two vertical tubes are presented in this section. Figure 5 shows the good agreement between experiments and numerical solutions for heat transfer coefficient in base flow without nanoparticles for different Reynolds number. Heat transfer coefficient is simply calculated from $q^{\prime \prime} /\left(T_{w}-T_{b}\right)$. The important parameter is $T_{b}$ which can be defined as: 
$T_{b}=\frac{\int \rho c_{p} V T d A}{\int \rho c_{p} V d A}$

$c_{p}$ is the fluid specific heat capacity.

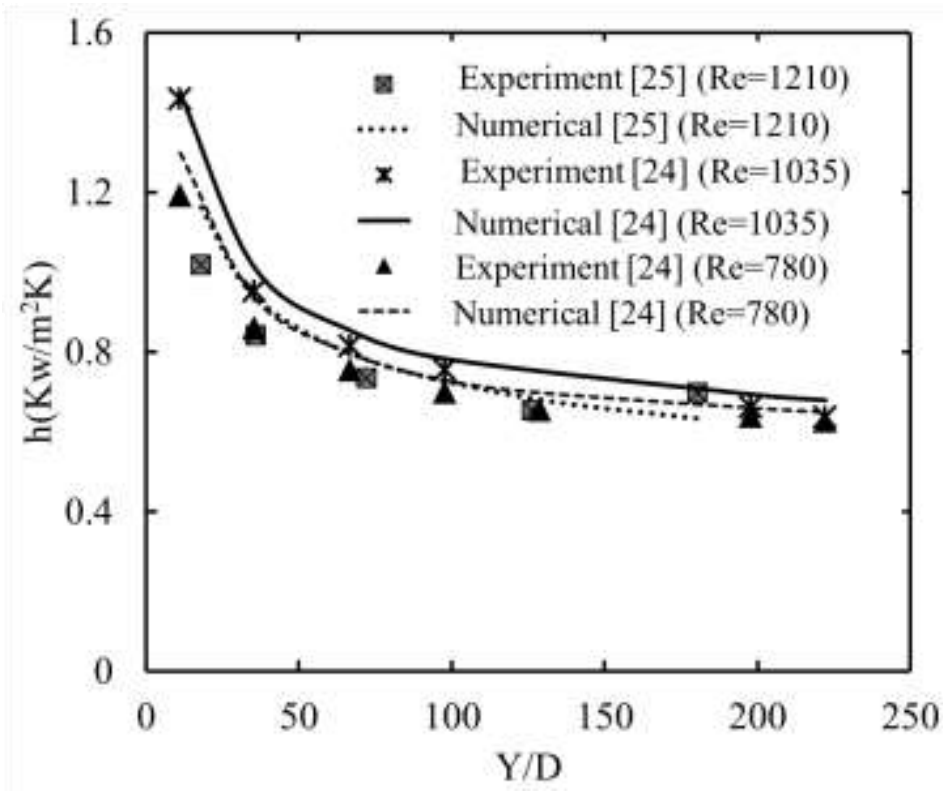

Figure 5. Heat transfer coefficient predicted by numerical solution and experimentally measured for laminar flow in vertical tubes

Heat transfer coefficient and $\mathrm{Nu}$ number estimated by Mixture model and DPM comparing to experimental results for three types of nanofluid are illustrated in Figure 6 and 7. A wide range of Reynolds number from 290 to 1800 and particle volume fraction from $0.2 \%$ to $2.76 \%$ has been simulated for three common nanofluids, i.e. Alumina, Zirconia and Silica nanofluid, with different diameter of nanoparticles. 

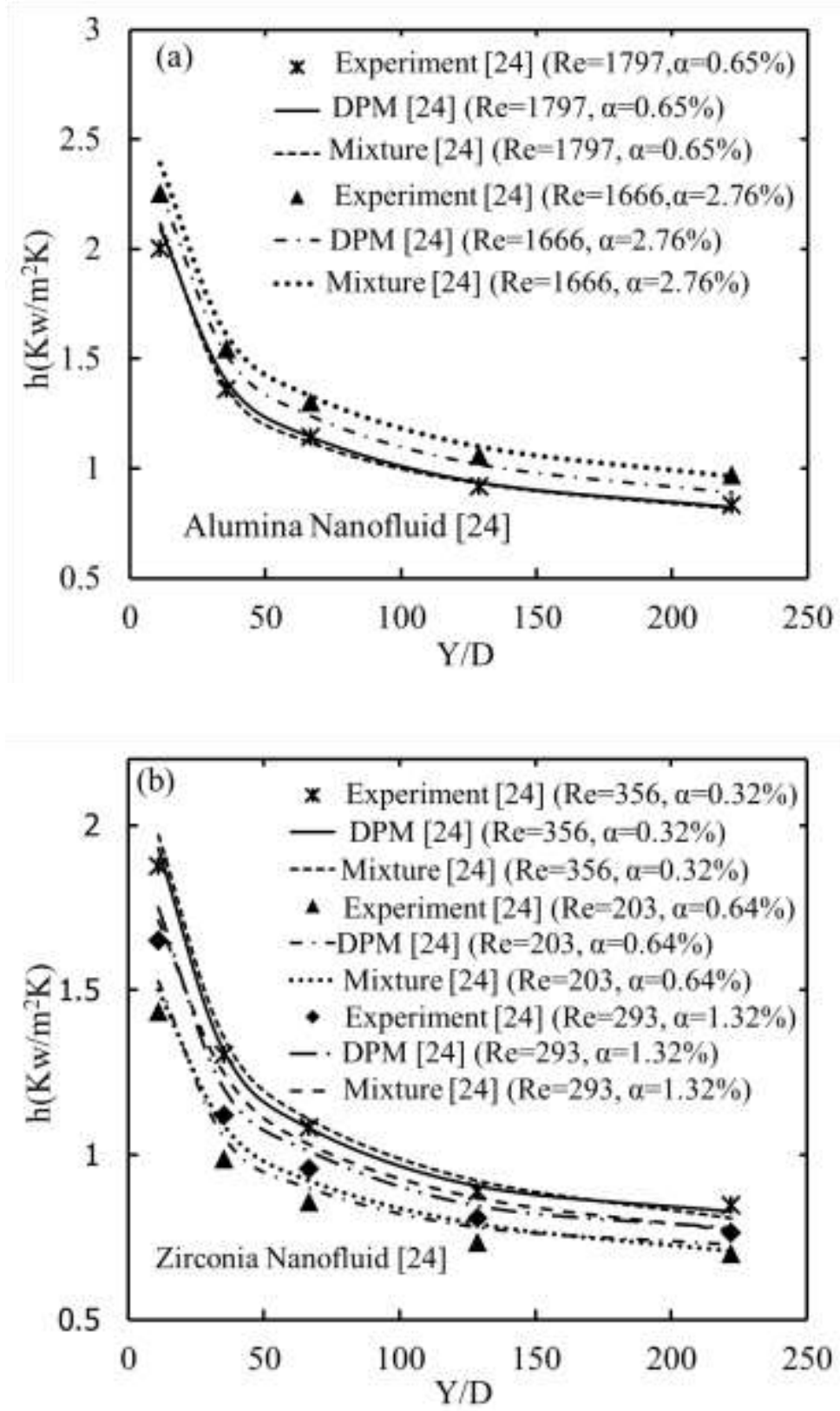

Figure 6. Comparison of experimental data and numerical predictions for heat transfer coefficient by Mixture model and DPM for a) Alumina b) Zirconia nanofluid.

It is clear that both models have provided findings in good agreement with experimental measurements. This conclusion is of importance, because empirical or theoretical correlations for thermo-physical properties of nanofluid are needed to simulate this sort of fluid in tubes. While DPM treats each component in nanofluid as it is, and only some general correlations like interaction or diffusion forces are required. As a result, DPM can be highly recommended for laminar nanofluid flow in vertical tubes to characterize thermal features of nanofluids. 


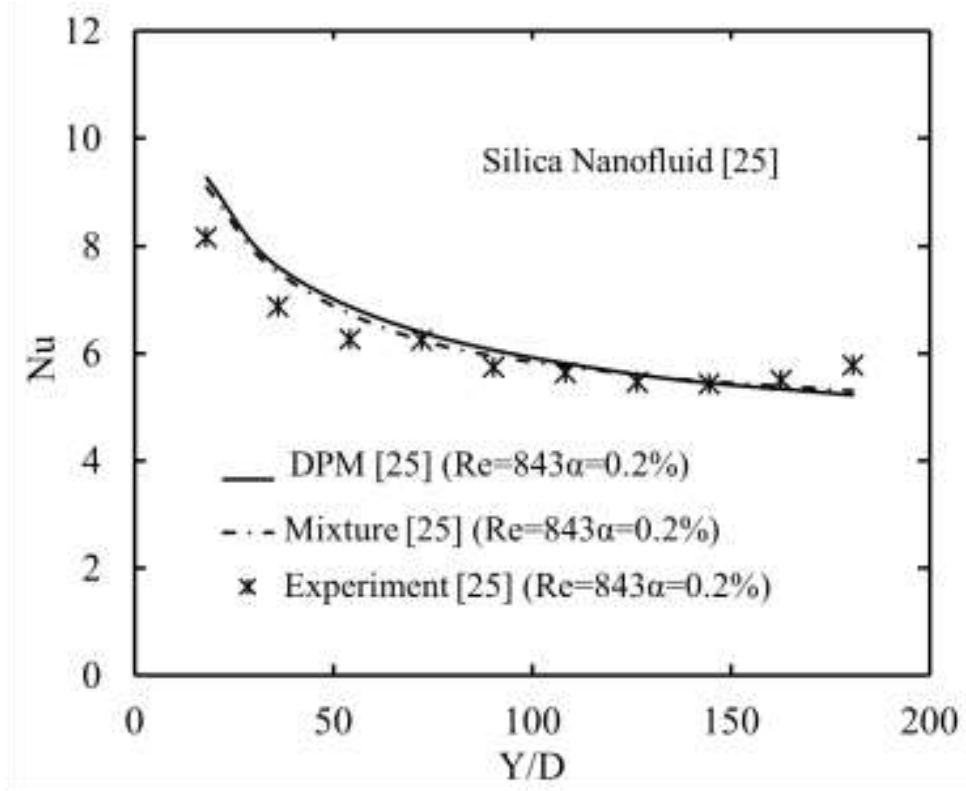

Figure 7. Nu number estimated by Mixture model and DPM in comparison with measured values for Silica nanofluid

As more explanation, the results in figures 6 and 7 are stated in terms of nondimensional length in vertical direction in figure 8 . Graetz number is defined as:

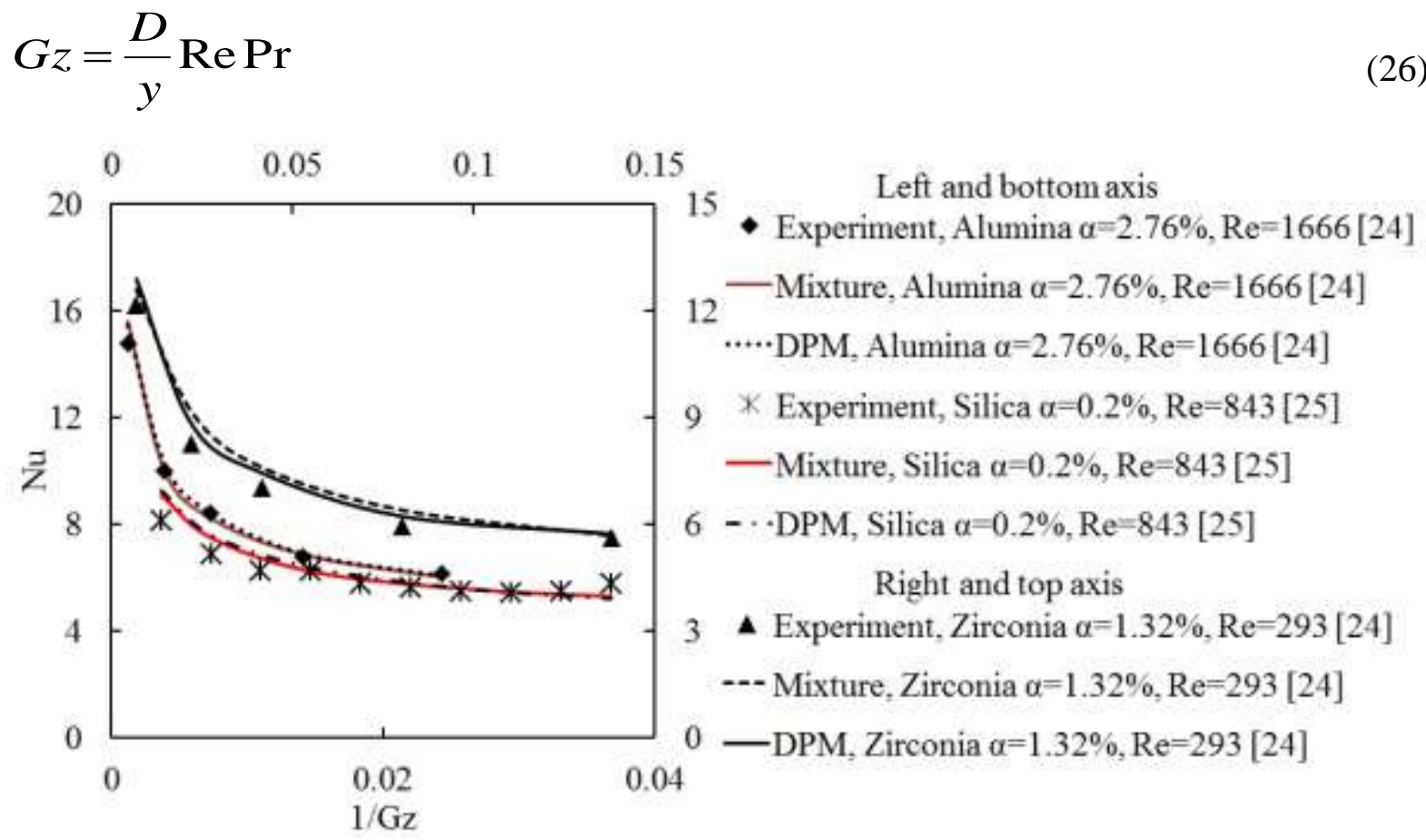

Figure 8. Local Nusselt number in terms of Graetz number in vertical direction 


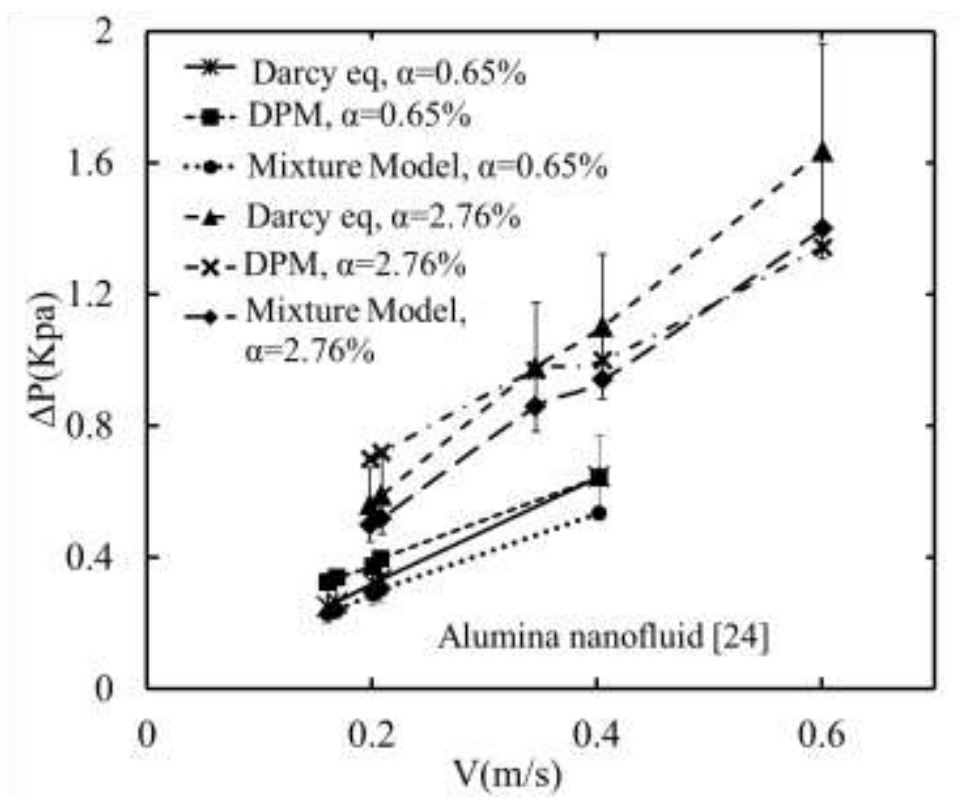

Figure 9. Prediction of pressure drops for Alumina nanofluid by Mixture model and DPM in comparison with the correlation

Pressure drops estimated by Mixture model and DPM are compared with the DarcyWeisbach correlation in the error range of $20 \%$ in Figures 9-11, presented as:

$$
\Delta P=\frac{64}{\operatorname{Re}} \frac{L \rho V^{2}}{2 D g}
$$

All the properties for Darcy-Weisbach correlation are borrowed from mixture properties presented in literatures. It has been explained that this correlation calculates pressure drops with maximum $20 \%$ error from measured data $[24,38]$. Whole the simulations were carried out for volume fraction below 3\% and a good agreement is observed between modelling and the correlation. DPM has shown better trend for pressure losses prediction comparing to Mixture model. The simulations were also performed for higher 3\% volume fractions. The DPM results were sometimes $100 \%$ different from the correlation, especially in the case of higher velocity shown and no matter the type of nanofluid. The main differences between lower and higher volume fraction can be introduced as: first, rising the number of nanoparticles in each computational cell. The major problem here can concern to the weakness of the weight or Gaussian function to distribute the effects of particle variables to neighbouring cells. Second, the possibility of clustering and collision among nanoparticles will expand with increase in volume fraction. 

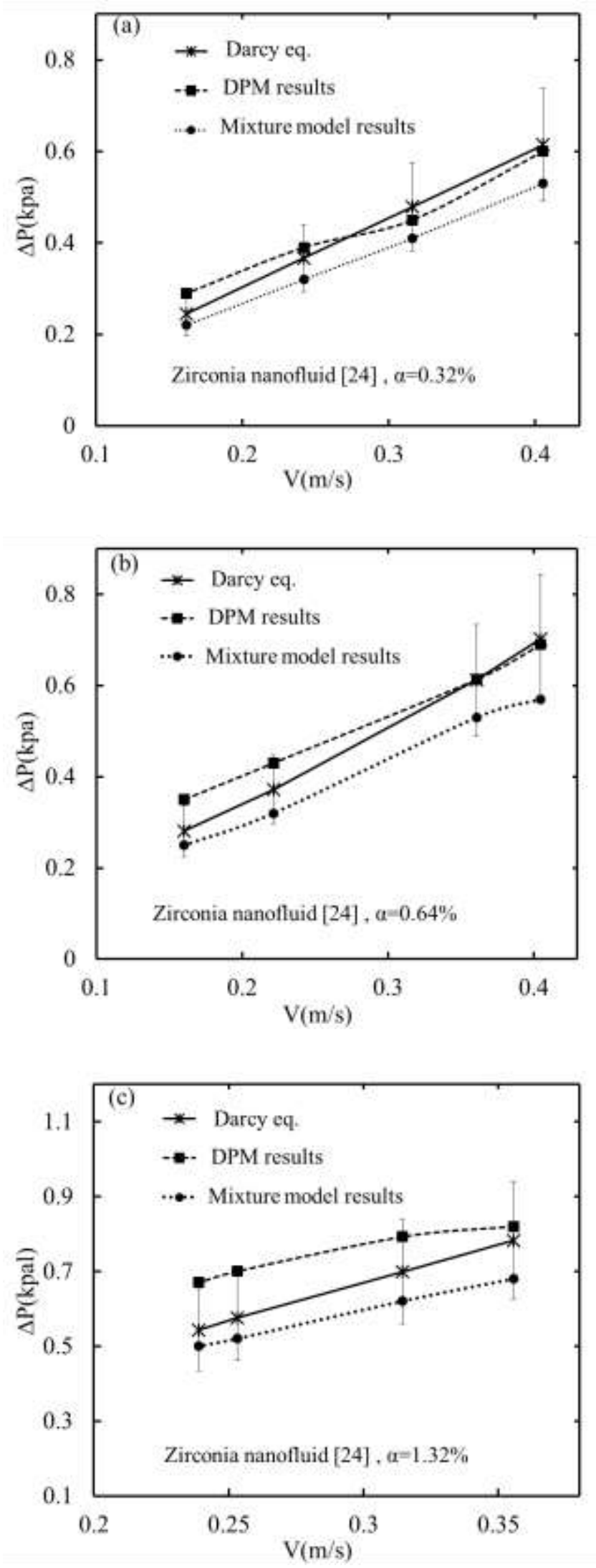

Figure 10. Prediction of pressure drops for Zirconia nanofluid by Mixture model and DPM in comparison with the correlation 
Due to higher number of Silica nanoparticles in each parcel comparing to other two nanoparticles with the same conditions, as shown in Figure 12, the percentage of difference for pressure drops in higher volume fraction were found much appreciable.
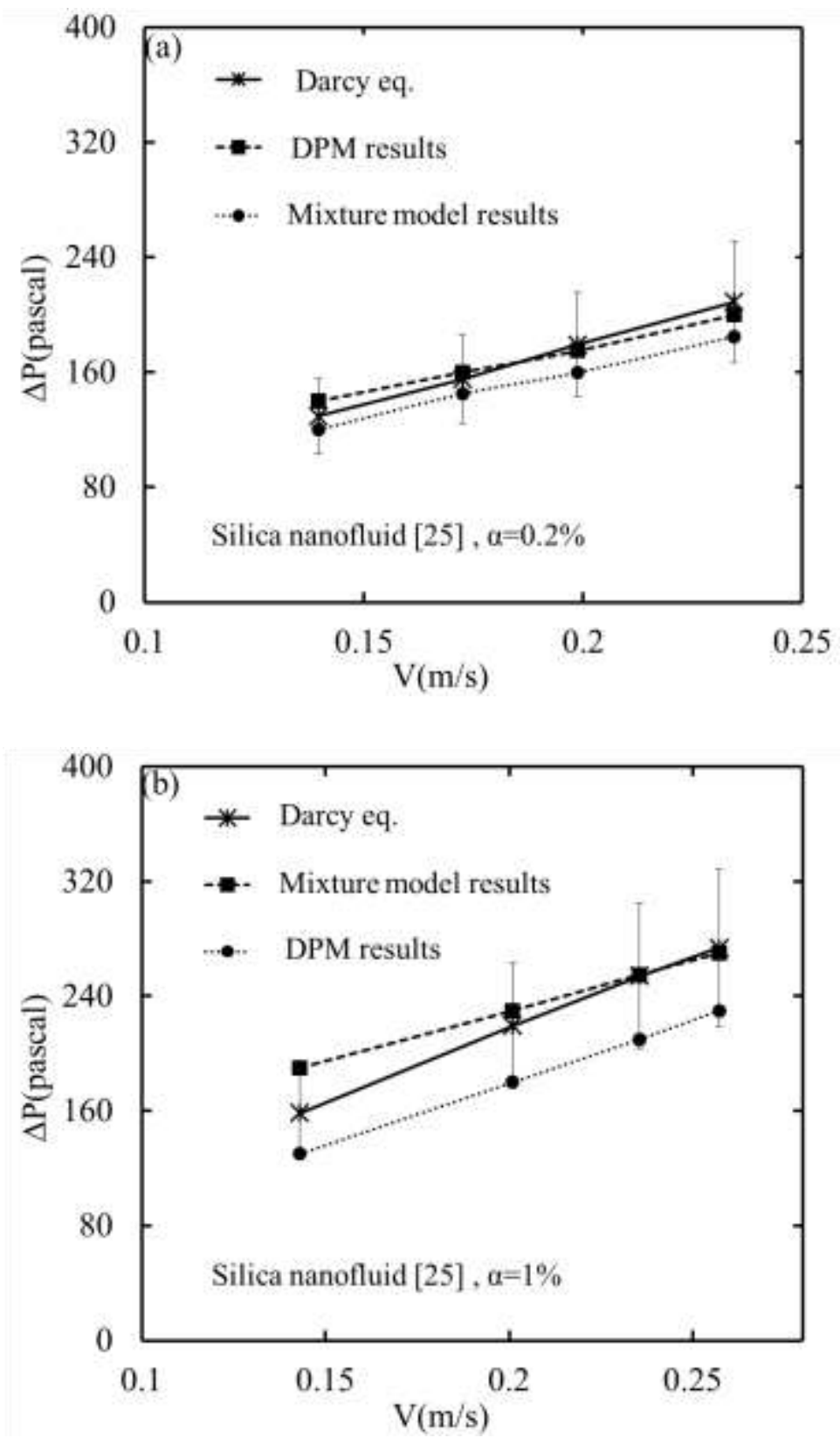

Figure 11. Prediction of pressure drops for Silica nanofluid by Mixture model and DPM in comparison with the correlation

It is noted that the number of particles are conserved in entire domain when they are solved in Lagrangian frame. It means that it is important to assign proper amount of the number of time step for nanoparticles to make sure all of the injected particles will leave the geometry model. 
Moreover, the average difference in total between simulations and Darcy equation for pressure drops was found near $12 \%$ by Mixture model and $10 \%$ by DPM.

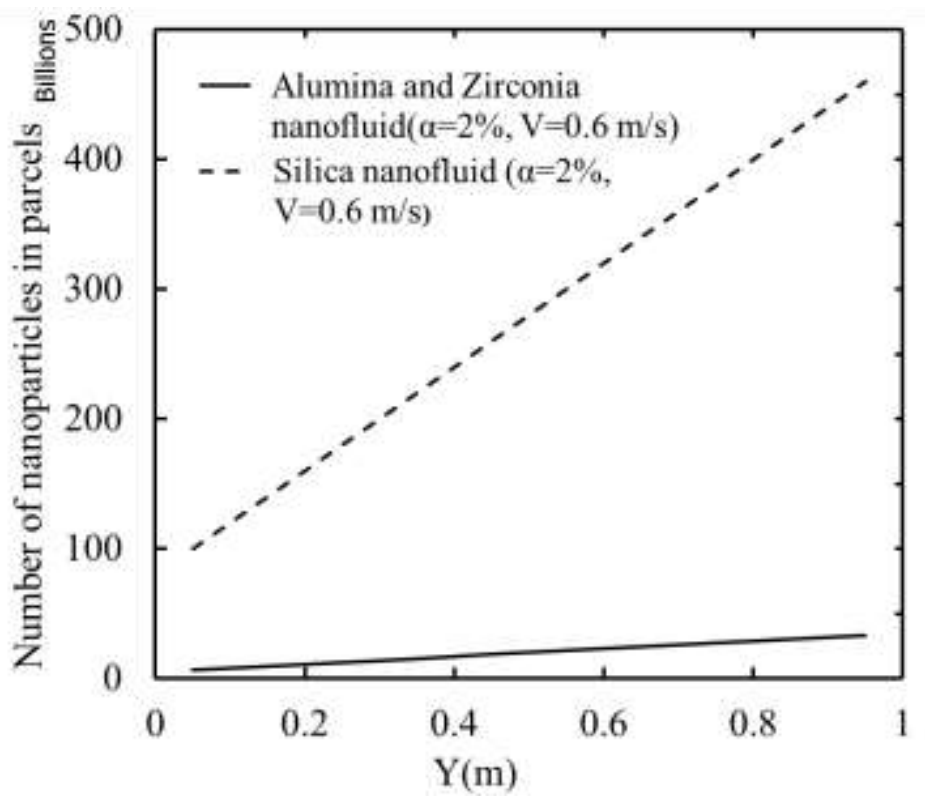

Figure 12. Changes in number of nanoparticle in each parcel from inlet to outlet at the center of the tube

Nondimensional temperature distribution of fluid and nanoparticles at the outlet is illustrated in Figures 13 and 14 for three nanofluids. With heat transfer parameters in this study consist of heat flux, temperature, thermal conductivity and characteristic length, non-dimensional temperature can easily be defined as:

$T^{*}=\frac{2\left(T-T_{i n}\right) \kappa}{q^{\prime \prime} D}$

$D$ is the inside diameter of the tube.

Smooth parabolic profile of temperature for base fluid is observed in all the simulations. It can be explained that nanoparticles are not able to distort the parabolic shape of temperature profile and energy is mostly transported via nanoparticle migration. This can be more sensed when fluid temperature is compared to distribution of nanoparticle temperature in Figures 13 and 14. The nanoparticle temperature profiles is distorted at the vicinity of the wall because of the higher temperature gradient. The trend is almost similar for lower and higher concentration. Although, the distribution of temperature seems more uniform in the case of Silica nanofluid with $0.2 \%$.

Variations of wall temperature by Alumina and Zirconia nanofluids achieved via two models are compared to a situation that fluid is not affected by nanoparticles in Figure 15 . The 
impacts of Zirconia nanoparticles on wall temperature seem small in the figure and the predictions of two models are close to the absence of nanoparticles. The gap will be more visible with increase in volume fraction from $1.32 \%$ in Zirconia to $2.76 \%$ in Alumina nanofluids. As can be expected, due to rise in transport of energy by nanoparticles, the wall temperature has reduced comparing to the fluid without particles as estimated well via both DPM and Mixture model. Also, Mixture model predicts more drops in wall temperature than DPM.

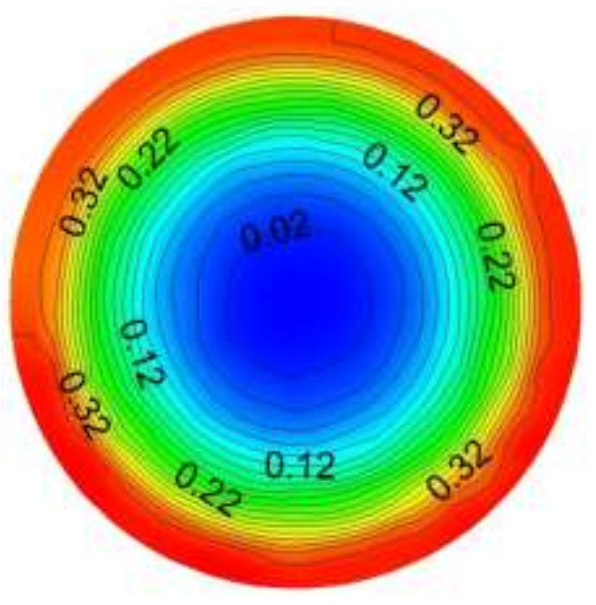

a) Alumina nanoparticle $(\alpha=0.65 \%$,

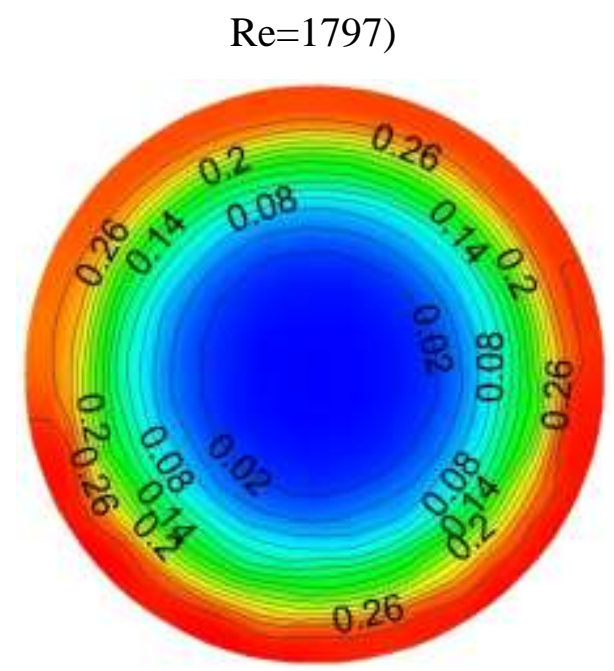

c) Alumina nanoparticle $(\alpha=2.76 \%$,

$$
\mathrm{Re}=1666)
$$

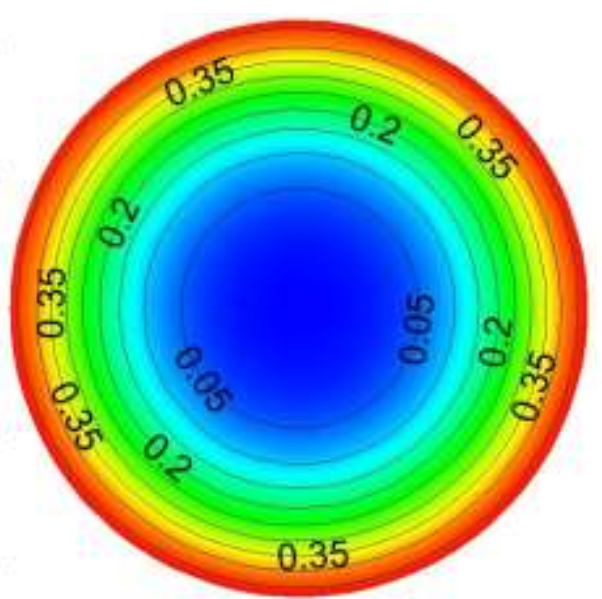

b) Base flow $(\operatorname{Re}=1797)$

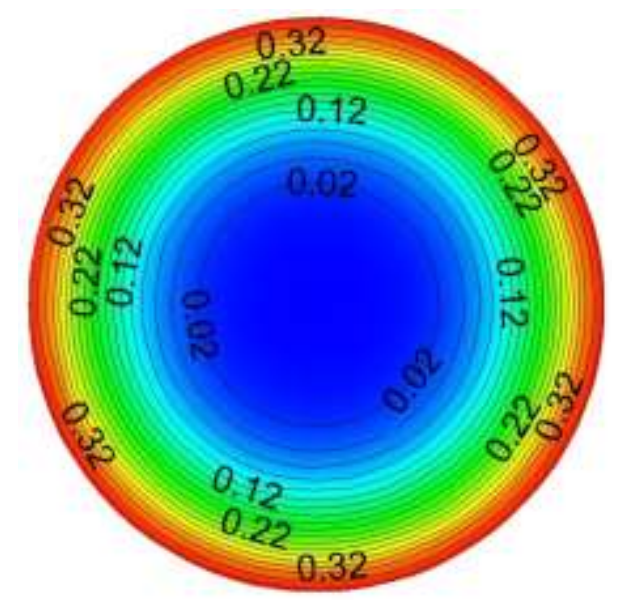

d) Base flow $(\operatorname{Re}=1666)$

Figure 13. Non-dimensional temperature distribution of base fluid and Alumina nanofluid predicted by DPM a and b) $T_{\text {in }}=21.7, q=18633 \mathrm{~W} / \mathrm{m}^{2}, c$ and d) $T_{\text {in }}=23.1, q=25306 \mathrm{~W} / \mathrm{m}^{2}$

Figure 16 presents velocity profile at the outlet of tubes on symmetry line predicted by two multiphase models. The model findings are indicated in comparison with the fluid velocity affected by nanoparticles. 


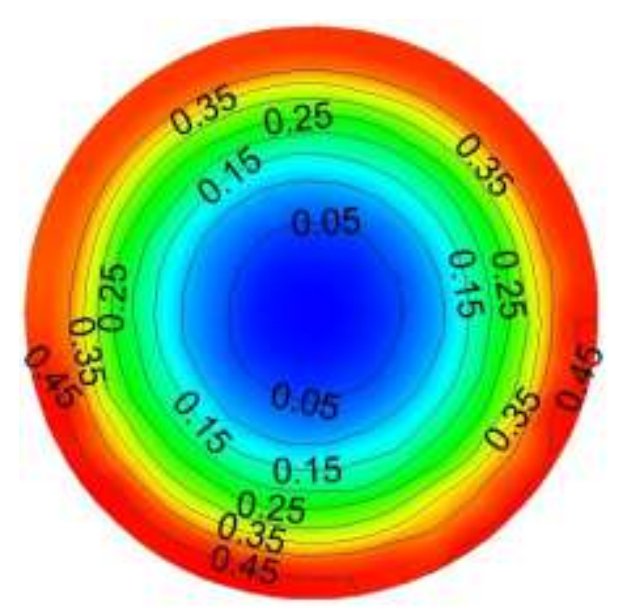

a) Zirconia nanoparticle $(\alpha=1.32 \%, \operatorname{Re}=293)$

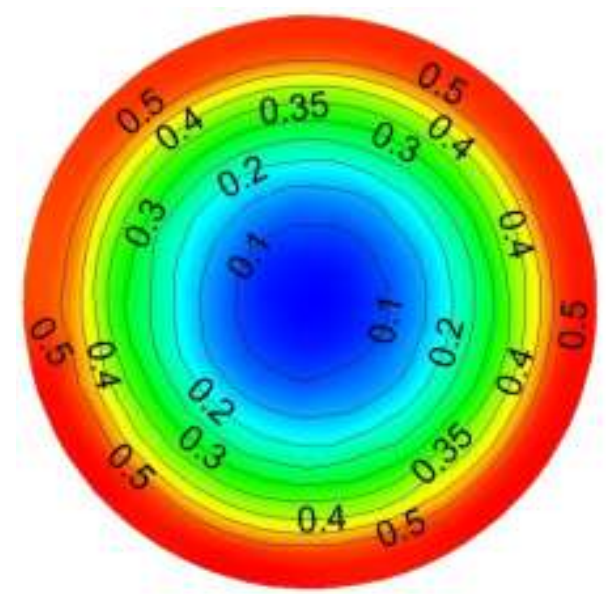

c) Silica nanoparticle $(\alpha=0.2 \%, \operatorname{Re}=843)$

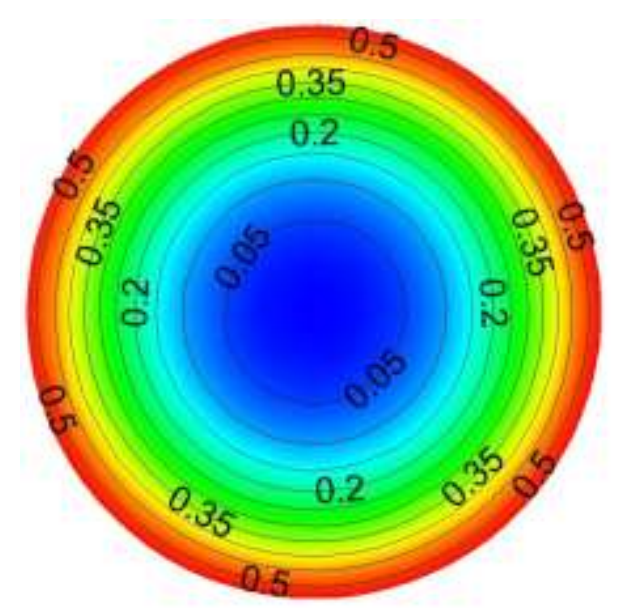

b) Base flow $(\operatorname{Re}=293)$

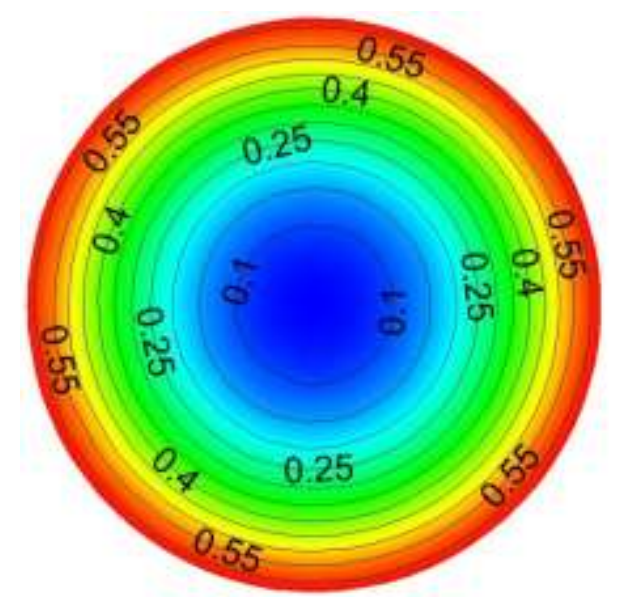

d) Base flow $(\mathrm{Re}=843)$

Figure 14. Non-dimensional temperature distribution of base fluid and nanofluid predicted by DPM a and b) $T_{\text {in }}=22, q=16308 \mathrm{~W} / \mathrm{m}^{2}, c$ and d) $T_{i n}=15, q=15765 \mathrm{~W} / \mathrm{m}^{2}$

The gap between particles and models velocity is largely seen close to the wall. The flow velocity profiles predicted by Mixture model and DPM are similar, while nanoparticles velocity profile shows small slip velocity with base flow. It is noted that due to high number of particles in the flow domain, this small amount of slip velocity is able to somehow produce noticeable pressure drops. From the figures, it seems that there is velocity for particles on the wall. As previously mentioned, boundary conditions for particles differs from fluid. No-slip condition holds only for fluid due to continuum which means zero velocity for fluid at the wall. The condition for particle is associated to collision between nanoparticles and wall (continuum is not held for dispersed particles). It means that if the nanoparticles do not reach the wall, the wall boundary conditions for particles are meaningless. On the other hand, since the nanoparticles are carried properly with fluid, simulations have revealed nanoparticles never hit the wall in this study. Hence, the unreal velocity is chosen for nanoparticles at the 
wall, which is equal to the cell velocity at the vicinity of the wall. However, this unreal velocity has no effects on modelling.

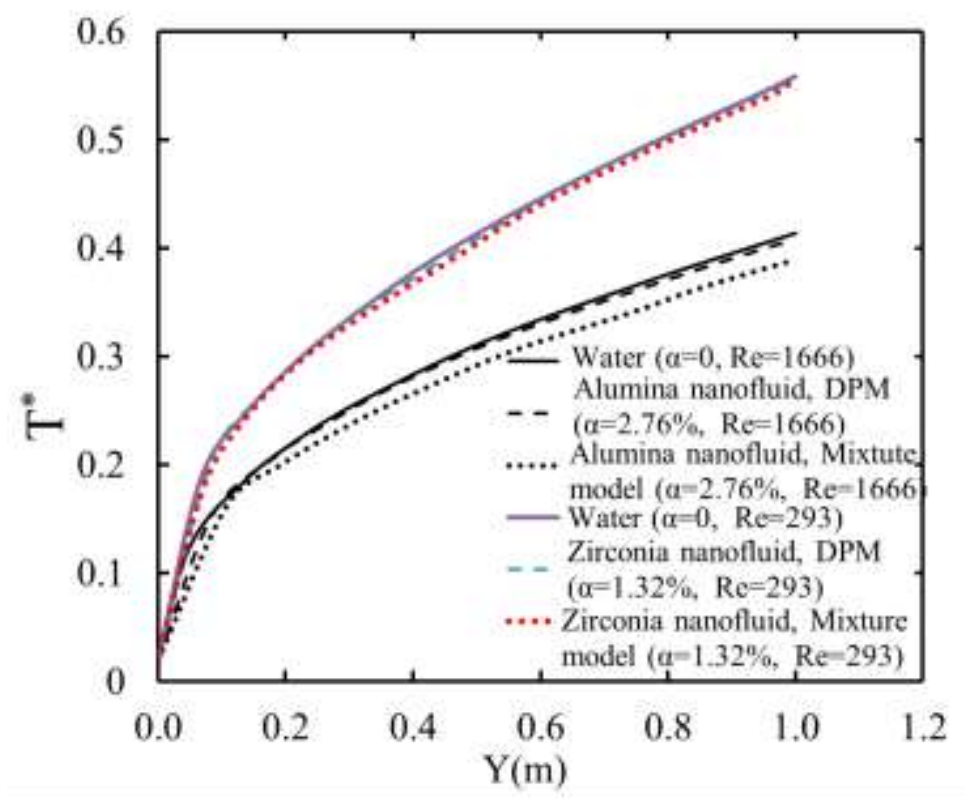

Figure 15. Longitudinal variations of non-dimensional wall temperature influenced by Alumina and Zirconia nanofluids.

\section{Conclusion}

Numerical simulation of heat transfer and pressure drops of nanoparticles inside water through a vertical tube in laminar flow was investigated and compared with experimental data available in the literature. Three types of nanoparticles with different diameters were considered as Alumina, Zirconia and Silica nanoparticles. Both Mixture model from Eulerian and Discrete Phase Model from Lagrangian approach were used to estimate the thermal and hydro-dynamic behaviours of nanofluids. It has been observed that using a proper weight function seems crucial to distribute the influences of particle variables to neighbouring's cells in DPM. For this aim, Gaussian kernel function was used. Heat transfer results showed that there is a good agreement between experiments and simulations for all the nanofluids in this study and various ranges of volume fraction from $0.2 \%$ to $2.76 \%$. Therefore, DPM can be highly recommended to model heat transfer in vertical tubes with laminar nanofluid flow. Furthermore, DPM deals with only thermo-physical properties of base fluid as opposed to Mixture model which requires thermal-fluid properties of nanofluids from empirical correlations. Pressure drops findings have revealed different trend than heat transfer simulations. They can be reliable only for volume fraction less than $3 \%$ for all of three types 
of nanofluids. Changes of volume fraction in outlet cross section are small and DPM could not report clustering of nanoparticles.
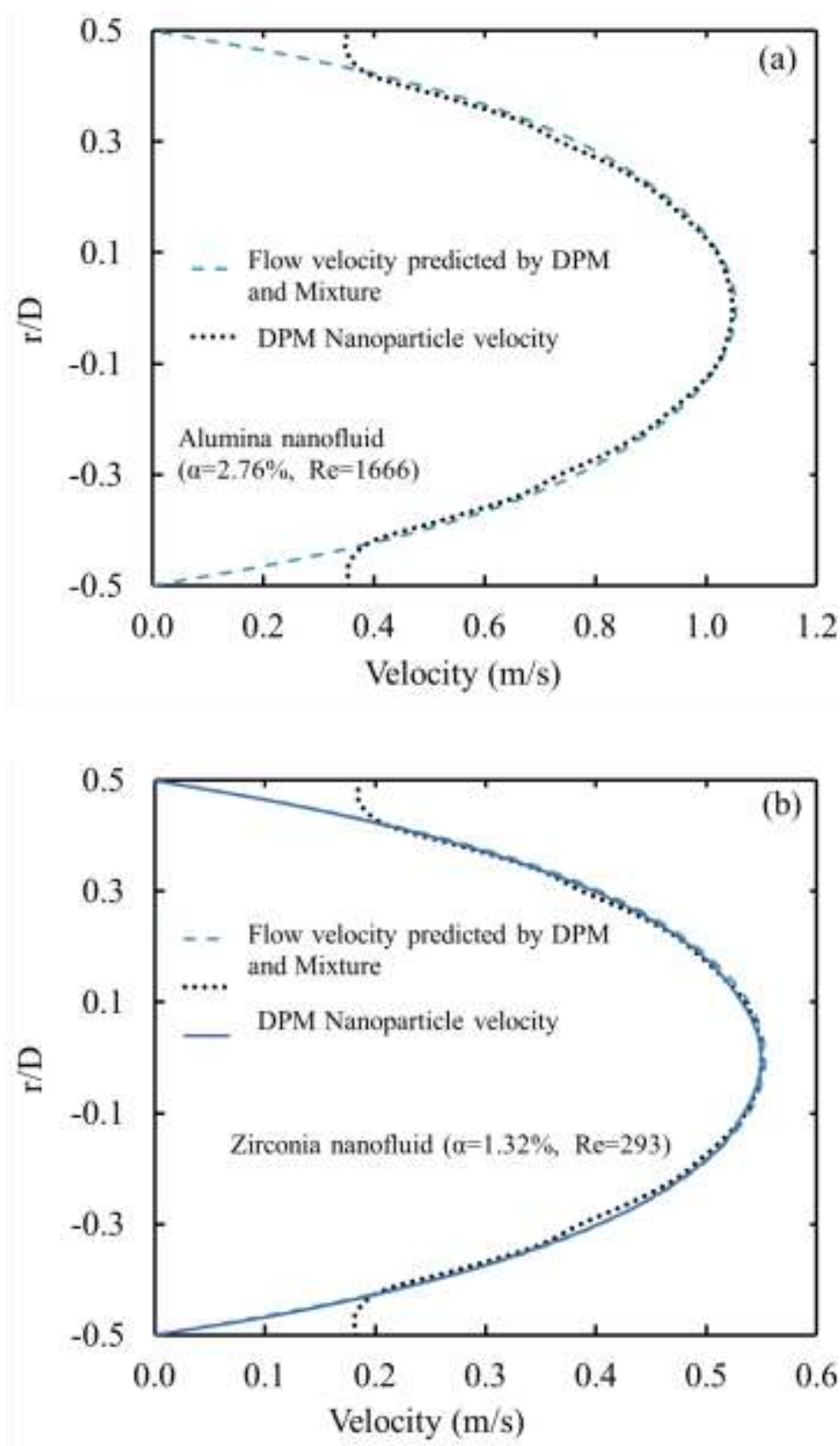

Figure 16. Velocity profile on the symmetry line at the outlet of tubes for Alumina, Zirconia and Silica nanofluids.

Mixture model presents the same velocity and temperature profile for both phases, while DPM has shown the ability of capturing slip velocity and temperature difference between particles and liquid. The smooth parabolic profiles of fluid temperature and velocity highlight that nanoparticle migration is one of the most significant phenomena in rise of heat transfer and energy transportation. 
Here are some comments about the benefit of DPM in laminar vertical pipe flow:

1. Mixture model results highly depend on transport properties coming from experimentation. There is no need the mixture properties for DPM and it only needs appropriate interaction or diffusion forces, empirical or analytical.

2. Mixture considers nanofluid as two phases. DPM behaves the nanofluid as fluid and solid, as it is in the reality.

3. Thermo-physical properties correlations for nanofluid are not universal and changes by fluid and nanoparticles. Empirical correlations for DPM interaction forces are not that extensive and can be employed in most the cases.

4. Effects of slip mechanism like Thermophoresis and Brownian can be seen as diffusion terms in Mixture equations and need to be implemented in program. In DPM, velocity is calculated for fluid and solid separately and eventually the relative velocity between them has the key role.

DPM is weak to simulate higher nanoparticle loading due to inclusion other phenomena such as clustering and aggregating.

\section{Nomenclature}

$\begin{array}{llll}\mathrm{A}_{\mathrm{p}} & \text { particle surface area } & Q_{s} & \text { energy source term } \\ B i & \text { Biot number } & \mathrm{Re}_{\mathrm{p}} & \text { Reynolds number } \\ C_{c} & \text { Cunningham correction } & \Delta t & \text { Lagrangian time step } \\ C_{D} & \text { drag coefficient } & T & \text { temperature } \\ c_{p} & \text { liquid specific heat } & T^{*} & \text { Non-dimensional temperature } \\ c_{p_{p}} & \text { particle specific heat } & & \\ d_{p} & \text { particle diameter } & \text { Greek symbols } & \\ D & \text { tube diameter } & \alpha & \text { volume fraction } \\ f_{d} & \text { drag function } & \mu & \text { viscosity } \\ F_{B_{i}} & \text { Brownian force } & \rho & \text { density } \\ F_{s} & \text { momentum source term } & \psi_{i} & \text { Gaussian random noise function } \\ G_{w} & \text { Gaussian function } & \tau_{m} & \text { mixture shear stress tensor } \\ G_{z} & \text { Graetz number } & \theta & \text { particle or parcel variable } \\ H & \text { enthalpy } & & \\ h & \text { heat transfer coefficient } & \text { Subscripts } & \\ \kappa & \text { thermal conductivity } & b & \text { bulk } \\ K_{B} & \text { Boltzmann constant } & c & \text { continues or liquid phase }\end{array}$




$\begin{array}{llll}m_{p} & \text { particle mass } & \text { in } & \text { tube inlet } \\ \dot{m} & \text { mass flow rate } & \text { in-cell } & \text { inlet of a cell } \\ N & \text { number of particles } & k & \text { phase } k \\ V_{k m}, V_{p m} & \text { drift velocity } & m & \text { mixture } \\ V_{\text {relative }} & \text { relative or slip velocity } & \text { out } & \text { tube outlet } \\ P r & \text { Prandtl number } & \text { out-cell } & \text { Outlet of a cell } \\ q, q^{\prime \prime} & \text { heat flux } & p & \text { particle }\end{array}$

\section{References}

1. K.S. Hwang, S.P. Jang, S.U.S Choi, Flow and convective heat transfer characteristics of waterbased $\mathrm{A} 12 \mathrm{O} 3$ nanofluids in fully developed laminar flow regime, International Journal of Heat and Mass Transfer 52 (2009) 193-199.

2. Y. Ding, H. Alias, D. Wen, R.A. Williams , Heat transfer of aqueous suspensions of carbon nanotubes (CNT nanofluids). International Journal of Heat and Mass Transfer 49 (2006) 240-250.

3. V.I. Terekhov, S.V. Kalinina, V.V. Lemanov, The mechanism of heat transfer in nanofluids: state of the art (review). Part 2. Convective heat transfer. Thermophysics and Aeromechanics 12 (2010) 157-171.

4. A.M. Husseina, K.V. Sharmac, R.A. Bakara, K. Kadirgama A review of forced convection heat transfer enhancement and hydrodynamic characteristics of a nanofluid, Renewable and Sustainable Energy Reviews 29 (2014) 734-743.

5. D. Wen, Y. Ding, Experimental investigation into convective heat transfer of nanofluids at the entrance region under laminar flow conditions. International Journal of Heat and Mass Transfer 47 (2004) 5181-5188.

6. D. Liu, L. Yu, L. Single-Phase Thermal Transport of Nanofluids in a Minichannel. Journal of Heat Transfer 133 (3) (2011) .

7. A.T. Utomo, E.B. Haghighi, A.I.T. Zavareh, M. Ghanbarpourgeravi, H. Poth, R. Khodabandeh, Björn Palm, A.W. Pacek, The effect of nanoparticles on laminar heat transfer in a horizontal tube. International Journal of Heat and Mass Transfer 69 (2014) 77-91.

8. J.E. Julia, L. Hernández, R. Martínez-Cuenca, T. Hibiki, R. Mondragón, C. Segarra, J.C. Jarque. 
Measurement and modelling of forced convective heat transfer coefficient and pressure drop of $\mathrm{Al}_{2} \mathrm{O}_{3}$ - and $\mathrm{SiO}_{2}$-water nanofluids. Journal of Physics: Conference Series 395 (2012).

9. S. Suresh, K.P. Venkitaraj, P. Selvakumar, M. Chandrasekar. Effect of Al2O3-Cu/water hybrid nanofluid in heat transfer. Experimental Thermal and Fluid Science 38 (2012) 54-60.

10. S. Göktepe, K. Atalık, H. Ertürk, Comparison of single and two-phase models for nanofluid convection at the entrance of a uniformly heated tube. International Journal of Thermal Sciences 80 (2014) 83-92.

11. M. Hejazian, M.K. Moraveji, A. Beheshti, Comparative study of Euler and mixture models for turbulent flow of A12O3 nanofluid inside a horizontal tube. International Communications in Heat and Mass Transfer 52 (2014) 152-158.

12. M, Akbari. Numerical And Analytical Study Of Nanofluids Thermal And Hydrodynamic Characteristics. PhD thesis, Sherbrooke University, Quebec, 2012.

13. M. Kalteh, A. Abbassi, M. Saffar-Avvala, J. Harting, Eulerian-Eulerian two-phase numerical simulation of nanofluid laminar forced convection in a microchannel. International Journal of Heat and Fluid Flow 32 (2011) 107-116.

14. R. Lotfi, Y. Saboohi, A.M. Rashidi, Numerical study of forced convective heat transfer of Nanofluids: Comparison of different approaches. International Communications in Heat and Mass Transfer 37 (2010) 74-78.

15. S.P. Jang, S.U.S. Choi. Role of Brownian motion in the enhanced thermal conductivity of nanofluids. Applied Physics Letters 84 (2004) 4316-4318.

16. P. Keblinski, S.R. Phillpot, S.U.S. Choi, J.A. Eastman. Mechanisms of heat flow in suspensions of nano-sized particles (nanofluids), International Journal of Heat and Mass Transfer 45 (2002) $855-863$.

17. Z. Xiao-Feng, G. Lei. Effect of multipolar interaction on the effective thermal conductivity of nanofluids. Chinese Physics 16 (2007) 2028-32.

18. E.R. di Schio, M. Celli, A. Barletta, Effects of Brownian Diffusion and Thermophoresis on the Laminar Forced Convection of a Nanofluid in a Channel. Journal of Heat Transfer 136 (2014) 022401, 1-10. 
19. P.N. Nwosu, J. Meyer, M. Sharifpur, Nanofluid Viscosity: A Simple Model Selection Algorithm and Parametric Evaluation. Computers \& Fluids 101 (2014) 241-249.

20. W. Yu, S.U.S. Choi, The role of interfacial layers in the enhanced thermal conductivity of nanofluids: A renovated Maxwell model. Journal of Nanoparticle Research 5 (2003) 167-171.

21. Y. He, Y. Men, Y. Zhao, H. Lu, Y. Ding, Numerical investigation into the convective heat transfer of $\mathrm{TiO} 2$ nanofluids flowing through a straight tube under the laminar flow conditions. Applied Thermal Engineering 29 (2009) 1965-1972.

22. V. Bianco, F. Chiacchio, O. Manca, S. Nardini, Numerical investigation of nanofluids forced convection in circular tubes. Applied Thermal Engineering 29 (2009) 3632-3642.

23. S. Tahir, M. Mital, Numerical investigation of laminar nanofluid developing flow and heat transfer in a circular channel. Applied Thermal Engineering 39 (2012) 8-14.

24. U. Rea, T. McKrell, L. Hu, J. Buongiorno. Laminar convective heat transfer and viscous pressure loss of alumina-water and zirconia-water nanofluids. International Journal of Heat and Mass Transfer 52 (2009) 2042-2048.

25. Z. Zhang, Experimental evaluation of heat transfer characteristics of silica nanofluid. PhD thesis, Massachusetts Institute Of Technology, Massachusetts, 2010.

26. G. S. McNab, A. Meisen, Thermophoresis in Liquids. Journal of Colloid and Interface Science 44 (1973) 339-346.

27. M. Ishii, T. Hibiki. Thermo-Fluid Dynamics Of Two-Phase Flow, second ed., Springer, 2006, pp. 103-108.

28. M. Manninen, V. Taivassalo, S. Kallio. On the mixture model for multiphase flow. VTT Publications 288, Technical Research Centre of Finland. 1996.

29. L. Schiller, Z. Naumann. A drag coefficient correlation. V.D.I. Zeitung 77 (1935) 318-320.

30. U. L. REA,. Experimental study of Alumina-water and Zirconia-water nanofluids convective heat transfer and viscous pressure loss in laminar regime. Bsc dissertation, Massachusetts Institute Of Technology, Massachusetts, 2008, pp. 24-25.

31. S.A. Morsi, A.J. Alexander. An investigation of particle trajectories in two-phase flow systems. 
Journal of Fluid Mechanics 55(1972) 193-208.

32. W.J. Minkowycz, E.M. Sparrow, J. Murthy. Handbook of Numerical Heat Transfer. second. ed. Wiley, Hoboken, 2006. p. 71.

33. Manual. FLUENT15. User. Fluent Incorporated, 2014.

34. J. Ferziger, H. Peric, Computational Methods for Fluid Dynamics. 3rd. ed. Springer Berlin Heidelberg, 2002.

35. S.V. Apte, K. Mahesh, T. Lundgren. Accounting for finite-size effects in simulations of disperse particle-laden flows. International Journal of Multiphase Flow 34 (2008) 260-271.

36. D. M. Snider, An incompressible three-dimensional multiphase particle-in-cell model for dense particle flows. Journal of Computational Physics 170 (2001) 523-549.

37 A. Kaufmann, M. Moreau, O. Simonin, J. Helie, Comparison between Lagrangian and mesoscopic Eulerian modelling approaches for inertial particles suspended in decaying isotropic turbulence. Journal of Computational Physics 277 (2008) 6448-6472.

38. W. Williams, J. Buongiorno, LiL.W.Hu. Experimental Investigation of Turbulent Convective Heat Transfer and Pressure Loss of Alumina/Water and Zirconia/Water Nanoparticle Colloids (Nanofluids) in Horizontal Tubes. Journal of Heat Transfer 130 (2008). 